\title{
Creencias y prácticas culturales de las madres sobre el cuidado del niño menor de cinco años en Tarata-Tacna
}

\section{Mother's cultural beliefs and care practices on children under five years old in Tarata-Tacna}

\author{
Karimen Jetzabel Mutter Cuellar ${ }^{1 \mathrm{a}}$ \\ Elena Cachicatari Vargas ${ }^{1 \mathrm{~b}}$ \\ ${ }^{1}$ Escuela Profesional de Enfermería de la Universidad Nacional Jorge Basadre Grohmann. Tacna, Perú. \\ ${ }^{a}$ Docente, Maestra en Ciencias con Mención en Salud de la Mujer, del niño y del adolescente. \\ ${ }^{\mathrm{b}}$ Docente, Doctora en Ciencias: Enfermería.
}

\section{RESUMEN}

Objetivo: Analizar a través de las manifestaciones, las prácticas y creencias culturales de la madre en el cuidado del niño menor de cinco años. El escenario tomó lugar en la provincia de Tarata de la Ciudad de Tacna. Los participantes fueron 10 madres de niños menores de cinco años. Material y métodos: La técnica fue la entrevista a profundidad y como instrumento una guía de entrevista semiestructurada, las cuales fueron grabadas y transcritas. La muestra se obtuvo por saturación con precedente de consentimiento informado. Resultados: En el análisis de la información se utilizó el método de teoría fundamentada mediante el proceso de codificación abierta, axial y selectiva en el que emergieron cinco categorías y ocho subcategorías, cuatro relacionadas a las creencias del cuidado y cuatro categorías relacionadas a las prácticas del cuidado, todas estas dieron origen a una categoría central "integración de saberes", la cual representa las creencias y prácticas que realizaron las madre en el cuidado del niño menor de cinco años. Conclusiones: La integración de saberes como categoría central nos indica que las creencias y prácticas del cuidado que realizan las madres actualmente en la Provincia de Tarata están relacionadas al empleo de conocimientos ancestrales utilizando la práctica de la medicina tradicional e integrando la medicina convencional haciendo uso de los servicios del establecimiento de salud de la localidad.

Palabras clave: Creencias, cuidado del niño, prácticas culturales.

\section{ABSTRACT}

Objective: To analyze through the mother's cultural manifestations, practices and beliefs about the care of the child under five years. The scenario took place in the Tarata province in Tacna. The participants were 10 mothers of children under five years old. Material and methods: The technique was the in-depth interview and as a tool a semistructured interview guide, which were recorded and transcribed. The sample was obtained by saturation with prior informed consent. Results: In the information analysis the method of grounded theory was used through the process of open, axial and selective coding in which five categories and eight subcategories emerged, four related to the beliefs of care and four categories related to the practices of care, all these gave rise to a central category "knowledge integration", which represents the beliefs and practices that the mother made in the care of the child under five years. Conclusions: The integration of knowledge as a central category indicates that the beliefs and practices of care currently performed by mothers in the Province of Tarata are related to the use of ancestral knowledge using the practice of traditional medicine and integrating conventional medicine using the services of the local health center.

Keywords: Beliefs, child care, cultural practices. 


\section{Introducción}

Las estadísticas sanitarias mundiales reflejan que 5,9 millones de niños menores de cinco años murieron en el 2014 por causas prevenibles, el $43 \%$ de ellos por enfermedades infecciosas, principalmente neumonía, diarrea y malaria. Además, se observó que el $25 \%$ de los niños del mundo tienen retraso del crecimiento; el 6,5\% tiene sobrepeso o son obesos; menos del $40 \%$ de los menores de un año recibe lactancia materna natural exclusiva hasta el sexto mes de vida y, por último, uno de cada tres niños (200 millones en todo el mundo) no logra realizar plenamente su potencial físico, cognitivo, psicológico y/o socioemocional debido a la pobreza, la mala salud, la nutrición suficiente, los cuidados y la estimulación insuficiente (1). En nuestro contexto, el Instituto Nacional de Estadística e Informática (INEI, 2012) nos indica que en el Perú aún persisten problemas que afectan la salud infantil como son las diarreas, el no cumplimiento de las coberturas en la vacunación, el bajo nivel educativo de las madres y la escasa capacidad económica (2). Esta problemática se observa sobre todo en las zonas rurales y de sierra estando relacionadas con las condiciones de vida, la contaminación ambiental y con patrones culturales de crianza. Al analizar estos datos observamos que existen factores de riesgo implicados en el desarrollo de los niños, siendo necesario entonces conocer las creencias y prácticas en el cuidado de los niños menores de cinco años relacionados a los procesos de salud enfermedad. Es importante, también, que el profesional de la salud tenga en cuenta la diversidad cultural del país conociendo a profundidad que prácticas de salud son consideradas por la comunidad en la que trabaja integrando un enfoque intercultural. El objetivo de este estudio fue analizar las prácticas y creencias culturales de la madre en el cuidado del niño menor de cinco años. El grupo cultural estuvo conformado por madres de niños menores de cinco años en la Provincia de Tarata del Departamento de Tacna, zona que se caracteriza por ser una región rural andina especialmente aymara. Esta Provincia es parte de la jurisdicción del Centro de Salud Tarata. La información se obtuvo del padrón nominal de los niños menores de cinco años que asistieron a los controles de crecimiento y desarrollo. Siendo un tipo de investigación cualitativa con metodología de teoría fundamentada, la que permitió describir los procesos que pueden explicar los fenómenos complejos de la experiencia cotidiana de los individuos. El propósito de esta investigación es contribuir con el desarrollo de la capacidad de proporcionar cuidados culturalmente congruentes. Asimismo, ayudar a los profesionales de enfermería y sobre todo a los que se desempeñan en el Programa de Crecimiento y Desarrollo a no sufrir choques o desencuentros culturales por desconocer y desaprovechar los saberes, y prácticas de la comunidad.

\section{Material y métodos}

La presente investigación es un estudio cualitativo de teoría fundamentada. Esta metodología permite utilizar un procedimiento sistemático cualitativo para generar una teoría que explique en un nivel conceptual una acción, una interacción o un área específica. Esta teoría es denominada sustantiva o de rango medio y se aplica a un contexto más concreto. El grupo cultural se determinó por la técnica de saturación y estuvo conformado por 10 madres de niños menores de cinco años perteneciente a la jurisdicción del Centro de Salud Tarata en la Provincia de Tarata del Departamento de Tacna en el periodo de marzo a abril del 2017. Los datos para realizar el seguimiento se obtuvieron del registro de niños que asistieron a los controles de crecimiento y desarrollo.

Se utilizó además el consentimiento informado por escrito para obtener la autorización de las madres participantes en el estudio. Se realizó la entrevista en el establecimiento de salud Tarata, en sus domicilios, y en la comunidad. Para la recolección de datos, la técnica fue la entrevista a profundidad y como instrumento una guía de entrevista semiestructurada. Las entrevistas fueron grabadas con autorización de cada una de las madres; además, se asignó a las participantes seudónimos como Madre 01, Madre 02, hasta Madre 10. Posteriormente, se transcribió y se realizó el análisis cualitativo de las entrevistas realizándose un microanálisis de las narrativas (línea por línea), identificándose los códigos iníciales. Luego, se formularon las categorías preliminares, realizando simultáneamente registros escritos del análisis para encontrar las propiedades en cada una de las categorías y, por último, se realizó la codificación selectiva, integrando los conceptos en torno a una categoría central, complementando con otras categorías y sub categorías necesarias para explicar la temática del estudio. Para comprender este fenómeno, los datos se interpretaron y organizaron en torno a la categoría central. Este proceso se inició con la transcripción de las entrevistas y la organización de las notas de campo realizadas. Con el objetivo de utilizar de manera óptima los datos, las entrevistas fueron transcritas por las investigadoras. El análisis de estos textos permitió a las investigadoras evidenciar como emergían los códigos y conceptos. La codificación y el análisis de datos se realizaron al finalizar la recolección de estos. Se hicieron entrevistas hasta agotar y saturar el muestreo teórico. Se construyó la teoría a través de la comparación constante entre las etapas del proceso de teorización. Cada entrevista contó con un número que la identificó, designado de acuerdo con el orden de surgimiento $(0,01,02,03$, etc.). Luego, se transcribió de manera textual cada entrevista grabada en medio de audio en un formato plano con el mismo tipo de letra y 
tamaño. En la generación de los códigos se etiquetaron las categorías según su surgimiento asignándoles letras y colores.

\section{Resultados}

El análisis que se realizó estuvo basado en la Metodológica de la Teoría Fundamentada. El procedimiento según Corbin y Strauss (3) implica una codificación abierta, una codificación axial y una codificación selectiva. Los pasos dentro del proceso de codificación fueron los siguientes:

1. Codificación abierta: En esta codificación el investigador revisa todos los segmentos del material para analizar y genera por comparación constante categorías iníciales de significado (4). En este proceso se identificaron dos categorías: creencias del cuidado y prácticas del cuidado. La primera se dividió en cuatro subcategorías: primero, niño sano; segundo, enfermedad, crecimiento y desarrollo; tercero, alimentos permitidos y, cuarto, diferencias entre niños y niñas. Ya la segunda se dividió también en cuatro subcategorías; primero, lactancia materna; segundo, alimentación; tercero, responsabilidad del cuidado y, cuarto, cuidado del niño enfermo.

2. Proceso de categorización: Según Corbin y Straus (4) una categoría representa un fenómeno, o sea, un problema, un asunto, un acontecimiento o un suceso que se define como significativo para los entrevistados. En ese proceso, se identifican las siguientes categorías: primero, visión del ser humano integral en sus esferas biopsicosocial; segundo, creencias populares; tercero, aplica la consejería de enfermería en sus prácticas del cuidado del niño; cuarto, la mujer cuida los hijos el hombre trabaja y, quinto, la práctica de medicina tradicional y medicina convencional.

3. Codificación axial: la codificación axial es el acto de relacionar categorías a subcategorías siguiendo las líneas de sus propiedades y dimensiones, así como de observar cómo estas se entrecruzan y vinculan (4). Una vez identificados dichos elementos, se realizó la agrupación por temáticas afines, permitiendo así la construcción de sub categorías y unidades de análisis del fenómeno.

4. Codificación selectiva: proceso de integrar y refinar la teoría (4). Al integrar las categorías principales se formó una categoría central para, finalmente, formar un esquema teórico mayor. La categoría central de la investigación es identificada como la "integración de saberes" representada en el modelo del sol naciente de Madeleine Leininger (7).

\section{Resultados}

Según el análisis realizado mediante la metodología de Teoría Fundamentada, en la Tabla 1, se identifica el proceso de codificación en el que se determinaron las categorías, subcategorías y unidades de análisis del estudio.

Tabla 1. Identificación de categorías, subcategorías y unidades de análisis

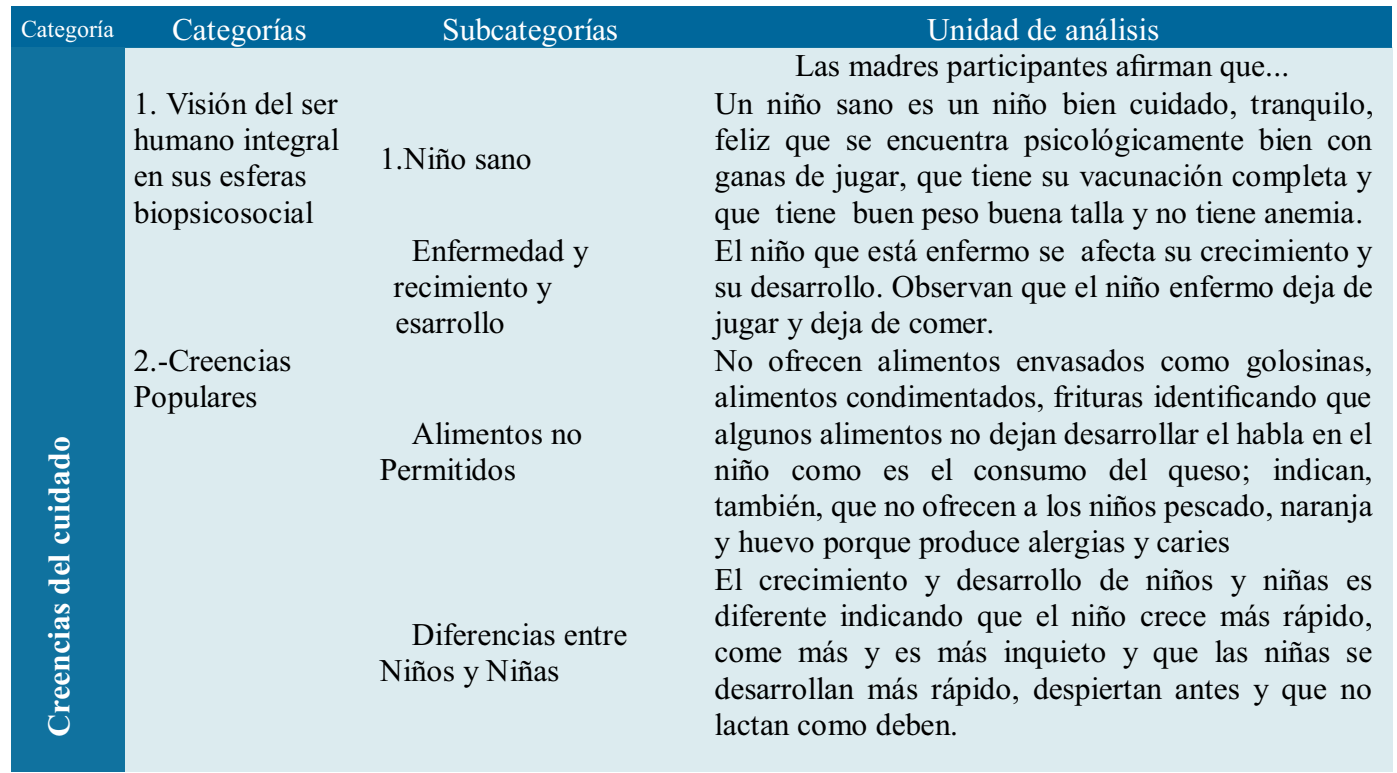




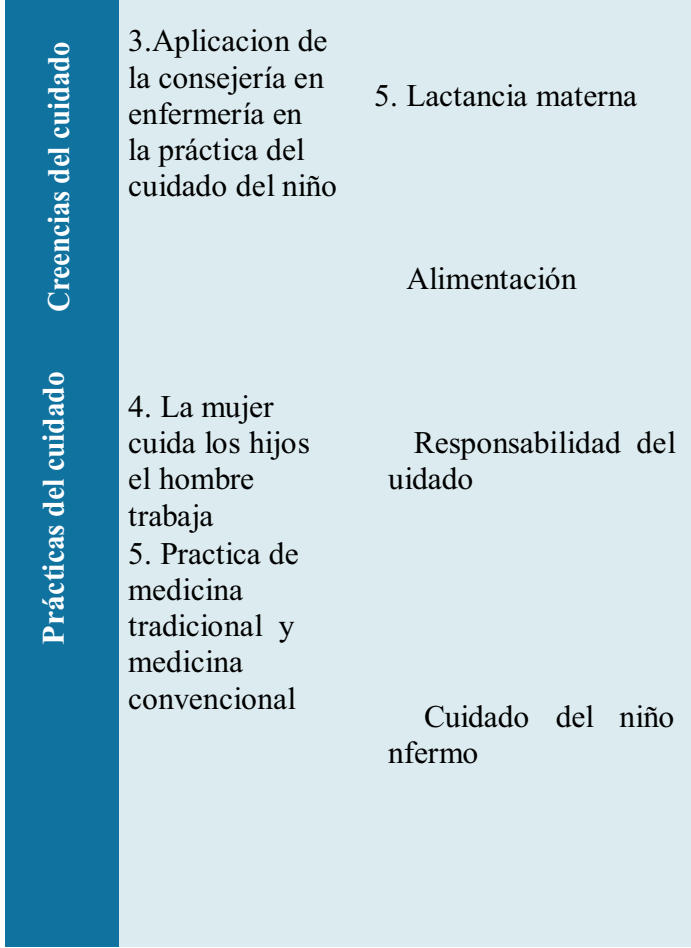

En la Figura 1 se observa la primera categoría denominada Creencias en el Cuidado. Las cual se subdivide en 02 subcategorías representadas como la visión del ser humano integral en sus esferas biospicosocial y las creencias populares de las madres de niños menores de 5 años de la provincia de Tarata.

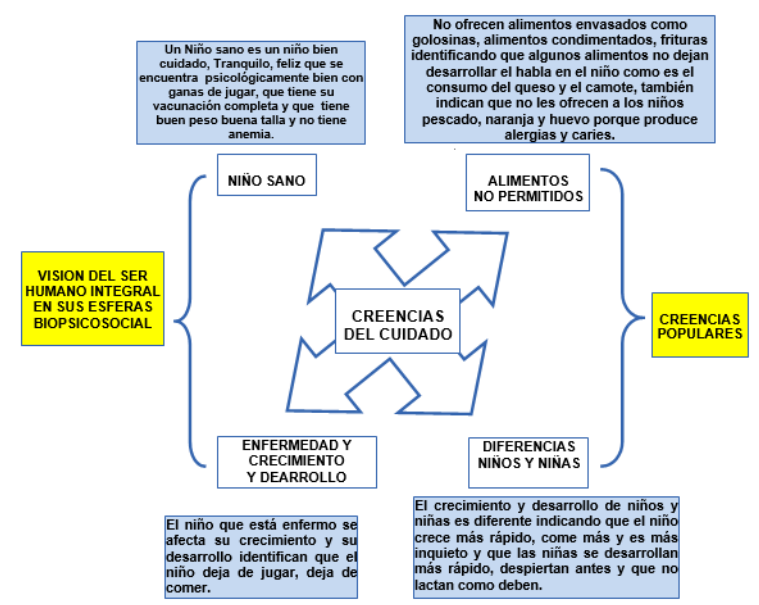

Figura 1. Categorías y subcategorías de las creencias en el cuidado del niño menor de cinco años.

En la Figura 2 se observa la segunda Categoría denominada Prácticas de cuidado. Las cual se subdivide en 03 subcategorías representada como la aplicación de la consejería en enfermería en las prácticas del cuidado del niño, prácticas de medicina tradicional y
La lactancia materna es exclusiva hasta los 6 meses y se debe dar al niño hasta los 2 años, es importante para el crecimiento y desarrollo del niño aumentando sus defensas, no siendo necesario el uso de fórmulas. $\mathrm{Al}$ inicio de la alimentación, integran los alimentos como el hígado, la sangrecita, la papa el camote la zanahoria el zapallo, menestras identifican que la consistencia al inicio es como papilla además muy pocas madres refieren el uso de la quinua, el huevo y otras leches.

El cuidado del niño está bajo la responsabilidad de la madre y el padre ayuda en el cuidado.

Identifican las enfermedades en dos grandes grupos las enfermedades respiratorias y las enfermedades digestivas, además de síntomas como la fiebre. Para estas enfermedades, utilizan la medicina tradicional entre las cuales se encuentran el uso de eucalipto, miel, limón, ruda, quico, orégano, leche materna, saliva, orina, manzanilla, grasa de pollo, frotación de mentol y aceite. Además, indican que se ayudan con la asistencia al establecimiento de salud. En caso de que se presente la enfermedad durante la noche algunas madres dan jarabes a sus hijos.

convencional y la subcategoría sobre la mujer cuida a los hijos y el hombre trabaja.

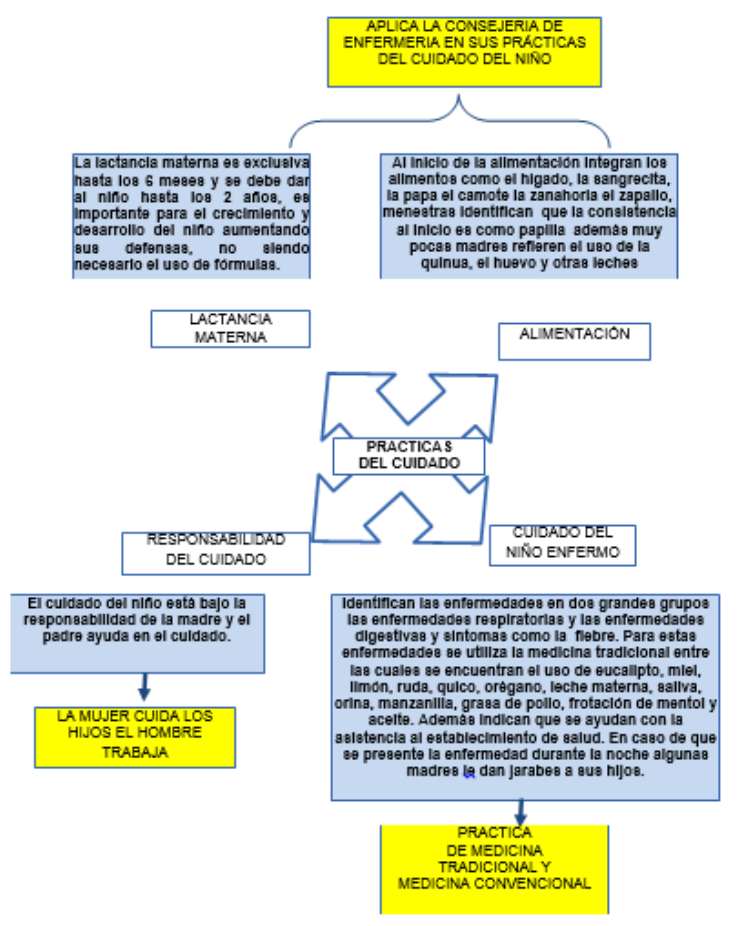

Figura 2. Categorías y subcategorías de las prácticas en el cuidado del niño menor de cinco años. 


\section{Discusión}

Interpretación y Teorización: Considerar las creencias y prácticas culturales relacionadas al cuidado del niño menor de cinco años de una población de la zona rural son observaciones que se han realizado en la práctica de enfermería al realizar las consultas de crecimiento y desarrollo a madres de zonas urbanas y rurales. Tales prácticas y cuidados fueron realizadas según costumbres y creencias locales.

Para la exposición de los resultados, se utilizó palabras de las madres participantes sobre la temática abordada.

\section{Explicación de la categoría central "Integración de} saberes": Esta categoría muestra que las creencias y prácticas del cuidado que realizan las madres en la zona rural alto andina de Tarata en Tacna, se realizan empleando conocimientos adquiridos de una generación a otra como es la práctica de la medicina tradicional a la vez que integran la medicina convencional haciendo uso de los servicios del Establecimiento de Salud de la localidad.

Esta categoría central se compone de las siguientes cinco categorías principales:
1.- Visión del ser humano integral en sus esferas biológica, psicológica y social.

2.- Aplicación de la consejería de salud en las prácticas del cuidado del niño.

3.- Practicas de medicina tradicional y medicina convencional.

4.- Creencias populares.

5.- Madre cuida, padre trabaja.

La Categoría Central se encuentra representada según el Modelo del Sol Naciente de Madeleine Leininger (7), en el que se determinan 4 niveles donde se encuentran representadas todas las categorias.

Nivel Uno: Visión del Mundo como las creencias y prácticas culturales.

Nivel Dos: Información de las madres: categoría central integración de saberes.

Nivel tres: Identificación de diversidad y universalidad de los cuidados, se determinan las categorías emergentes.

Nivel Cuatro: Visión de los cuidados culturales. Se identifican las categorías y subcategorías en los cuidados del niño menor de cinco años..
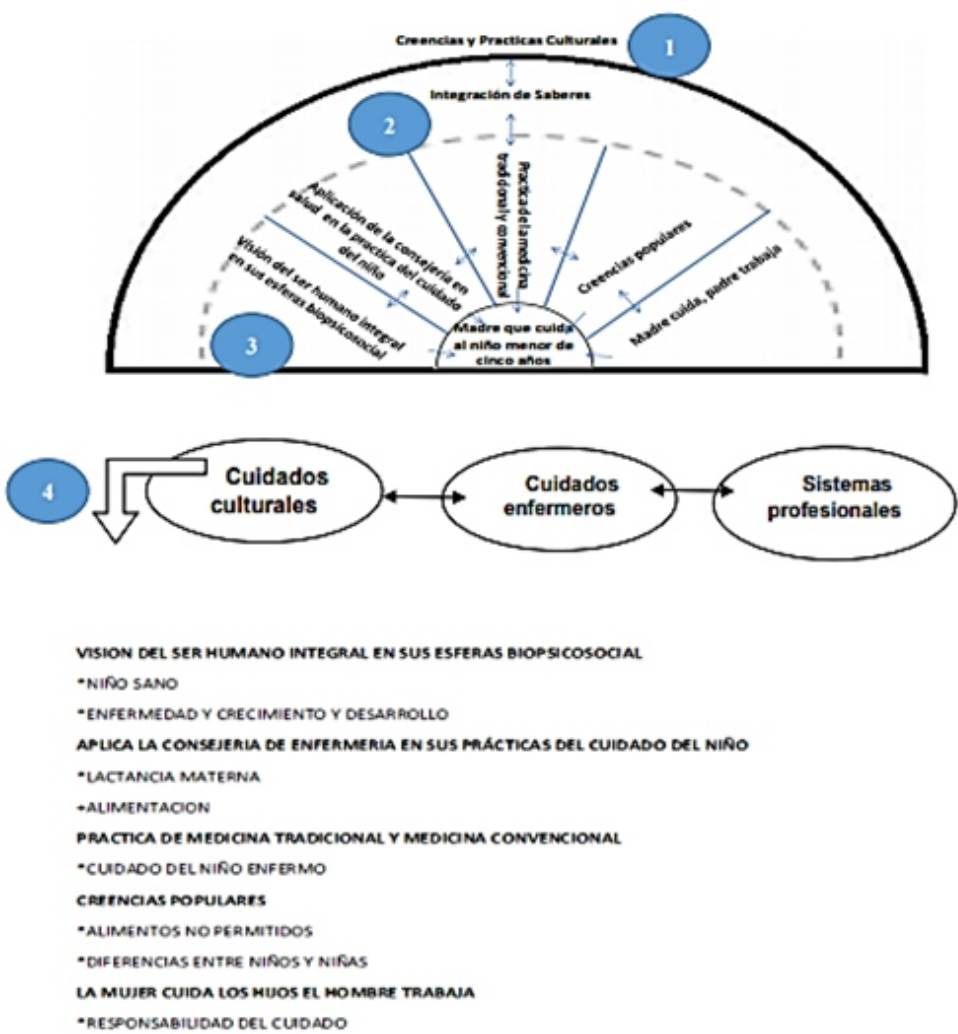

Figura 3. Modelo del sol naciente de Madeleine Leininger, adaptado para representar las categorías de las creencias y prácticas culturales en el cuidado del niño menor de cinco años en Tarata-Tacna, 2017. 


\section{2.- Descripción de las categorías}

La descripción de las categorías y sus correspondientes significados son los siguientes:

\subsection{Visión del ser humano integral en sus esferas biológica, psicológica y social}

Según Prieto (6) las creencias populares indican las actitudes de compromiso que mantienen los cuidadores ante determinados hechos concretos que se consideran dignos de una aceptación verdadera. Es decir, las prácticas que la familia brinda a los lactantes, o sea, esas creencias que mantienen los saberes populares ante enfermedades son consideradas por ellos como dignas y verdaderas (6). Leininger (7) plantea que este conjunto de valores, estilos de vida, creencias y normas que son aprendidas y compartidas, guían las acciones como un patrón intergeneracional.

La visión del ser humano integral en sus esferas biológica, psicológica y social está integrada por las subcategorías de niño sano y enfermedad crecimiento y desarrollo.

Las madres describen a un niño sano como:

Un niño sano es un niño bien cuidado,

tranquilo, feliz, que se encuentra psicológicamente bien, con ganas de jugar, que tiene vacunación completa y que tiene buen peso, buena talla y no tiene anemia.

Fuente: Mutter K. Entrevista Audio 02. Tarata-Tacna, 2017

Esta descripción pone de manifiesto que se considera la salud del niño como un equilibrio entre la parte física, psicológica y social. La madres entrevistadas destacan el aspecto emocional siendo las palabras más utilizadas por todas un niño tranquilo y feliz como indicador de buena salud. Según el ASIS del Pueblo Aymara en Puno en el 2010 en los talleres realizados, se señalaron como descriptores individuales de la salud para el pueblo Aymara: estar alegre, feliz, contento, con fuerza para trabajar, tranquilo sin preocupaciones $(8)$.

\section{2-Aplicación de la Consejería de enfermería en las prácticas del cuidado}

Las comunidades aymara siempre han sabido que la leche materna es fundamental para el desarrollo de la wawa. Por eso, la lactancia se prolonga por mucho tiempo, hasta que la wawa esté preparada física y emocionalmente para el destete. Tradicionalmente, el destete coincide con el primer corte de pelo de la wawa, ceremonia que marca su incorporación a la vida comunitaria" (9). En la narrativa de las madres sobre la lactancia materna, esta es identifican como parte de los cuidados que recibe el niño. El significado que le otorgan tiene relación a la educación que reciben en el establecimiento de salud utilizando los mensajes que allí aprenden. Las madres tienen interiorizada la importancia de lactancia materna como alimento fundamental para sus hijos e identifican el periodo de tiempo que deben otorgarle y la importancia frente a la enfermedad; asimismo, reconocen que las leches en polvo, para ellas, no contienen los nutrientes necesarios para sus hijos.

A continuación narrativas que representan esta categoría
"Siempre debes darle de lactar a un niño para que crezca sano y saludable”. Fuente: Mutter K. Entrevista Audio 02.Tarata-Tacna., 2017
"Es importante porque eso ayuda a crecer sanito, no es como la leche en polvo que se da, es un alimento primordial para nuestros hijos, es lo que se debe dar y no la leche en polvo".
Fuente: Mutter K. Entrevista Audio 09. Tarata-Tacna., 2017
"Para mí es lo básico porque las formulas no cumple con los nutrientes que dan las vitaminas de la lactancia mi hija tomo hasta los 2 años".
Fuente: Mutter K. Entrevista Audio 04.Tarata-Tacna., 2017

\section{3.- Prácticas de medicina tradicional y medicina convencional. \\ Con respecto al concepto de prácticas del} cuidado de la salud en el contexto cultural en que se encuentra la persona, Benner (10) afirma que los seres humanos están en una constante refinación de prácticas en los diferentes contextos, además estas constituyen una fuente de innovación.

Harris (11) indica la relación dinámica entre creencias y prácticas, las creencias influyen en las prácticas y estas a su vez pueden modificar las creencias. Entendemos entonces que tanto las creencias como las prácticas emergen de la realidad misma que rodea a las personas y que se utilizan para adaptarse o responder a su entorno. Entonces las prácticas de cuidado se desarrollan y siguen desarrollándose según características particulares, tales como el género, la ocupación y el contexto cultural en que se desarrollan las personas.

La medicina tradicional es todo el conjunto de conocimientos, aptitudes y prácticas, las cuales están basadas en teorías, creencias y experiencias indígenas de las diferentes culturas, sean o no explicables, usados para el mantenimiento de la salud, así como para la prevención, el diagnóstico, la mejora o el tratamiento de enfermedades físicas o mentales (13). A continuación se presentan narrativas en la que se identifica las prácticas de medicina tradicional y medicina convencional.

\footnotetext{
"Mi papá, como son a la antigua, me dice que cuando tiene diarrea o dolor de estómago le dé mate de ruda o mate de orégano o de anís y
} 
cuando tiene tos, mate de eucalipto, pero voy también a la posta para apoyarme",

Fuente: Mutter K. Entrevista Audio 05.Tarata-Tacna., 2017

"Cuando esta estreñido hacer tomar agua de anís, prácticamente yo hago lo que las enfermeras me dicen, porque mi mamá no está, porque no se mucho".

Fuente: Mutter K. Entrevista Audio 09.Tarata-Tacna., 2017

"Primerito si tiene diarrea yo le doy mate de quico, le froto los piecitos las plantas de abajo porque a veces por el frío le da, también le pongo medias, y le froto con manzanilla con aceite, cuando tiene enfermedad de la gripe le froto con mentol su pechito y después mate de eucalipto con miel, pero voy a la posta para apoyarme".

Fuente: Mutter K. Entrevista Audio 07.Tarata-Tacna., 2017

Se identifica que la población de estudio utiliza plantas medicinales con propiedades curativas en forma de infusión, baños, frotación. Estos conocimientos han sido adquiridos y trasmitidos de generación en generación de padres a hijos. El grupo de madres entrevistadas cuentan con un seguro integral de salud, y tienen acceso al establecimiento de salud.

La Organización Mundial de la Salud desde 1977 adoptó una resolución (WHA.30.49) (14). Lanzando una promoción mundial de la medicina tradicional, dicha resolución insta a los gobiernos miembros a dar importancia a sus sistemas médicos tradicionales. En 1978 se resaltó la importancia de las plantas medicinales en los sistemas médicos en países en desarrollo. La Medicina Tradicional es una parte importante y con frecuencia subestimada de la atención de salud (OMS 2013).

\subsection{Creencias populares}

Existe la creencia que algunos alimentos como el queso, no permite el desarrollo del lenguaje en los niños, Las creencias sobre el crecimiento y desarrollo de los niños pasan de generación en generación sin que muchas veces este tenga fundamento. Las características progresivas del desarrollo del lenguaje verbal en los diferentes niveles de edad, se adscriben a las etapas del desarrollo integral del niño, asociado a aspectos de maduración del sistema nervioso, al desarrollo cognoscitivo y al desarrollo socioemocional (15). Se puede decir que no todos los niños desarrollan las habilidades del habla y el lenguaje de la misma manera. Sin embargo, todos los niños siguen una progresión natural o una serie de etapas para dominar las habilidades del lenguaje (16).

Investigadores patrocinados por el Instituto Nacional de la Sordera y Otros Trastornos de la Comunicación USA (2010) han descubierto una variante genética vinculada específicamente al trastorno específico del lenguaje, un trastorno que retrasa el uso de palabras en el niño y disminuye el ritmo de dominación las habilidades del lenguaje durante la edad escolar. Este hallazgo es el primero que relaciona la presencia de una mutación genética específica con un tipo de deficiencia hereditaria en el lenguaje.

Otras investigaciones exploran el papel que podría desempeñar esta variante genética en la dislexia, el autismo y los trastornos del habla y la audición. Actualmente no se han encontrado evidencias que indiquen que algún alimento produzca un retraso en el lenguaje, identificándose que solo es una creencia que no tiene un fundamento científico.

A continuación narrativas que representan esta categoría

"No hay alimentos que perjudiquen a un niño todo pueden comer aunque hay creencias que el queso y el camote no le deja hablar al niño". Fuente: Mutter K. Entrevista Audio 03.Tarata-Tacna., 2017

"Algunas frutas, pero siempre le he dado queso como algunos dicen que el queso no le deja hablar".

Fuente: Mutter K. Entrevista Audio 06. Tarata-Tacna., 2017

"El huevo que le produce alergia, la naranja que los dientes sale caries" Fuente: Mutter K. Entrevista Audio 10.Tarata-Tacna., 2017

\section{5.- Madre cuida, padre trabaja}

El rol de la madre en la actualidad es dividir las tareas entre el cuidado de los hijos y el trabajo. En la zona alto andina de Tarata observamos que muchas de ellas se dedican al cuidado exclusivo de sus hijos y es el hombre el que realiza el trabajo pesado en las actividades de agricultura y ganadería aunque la mujer ayuda en estas labores, realizando pequeñas tareas mientras el hijo es lactante.

A continuación narrativa que representan esta categoría

\footnotetext{
"Yo cuido a los hijos y me ayuda mi esposo, solo cuando salgo algún sitio se los dejo a mi mama"

Fuente: Mutter K. Entrevista Audio 08.Tarata-Tacna., 2017
}

\section{Conclusiones}

Se concluye que las creencias culturales de las madres sobre el cuidado del niño menor de cinco años se identifican en las categorías visión integral del ser humano en sus esferas biológica, psicológica y social, entendiéndose esta como el significado que las madres dan a un niño sano como un niño bien cuidado, tranquilo y feliz, que se encuentra psicológicamente bien con 
ganas de jugar, que tiene su vacunación completa y que tiene buen peso, buena talla y no tiene anemia. Además, consideran que si el niño está enfermo se afectara su crecimiento y desarrollo identificando que el niño deja de jugar y de comer.

En relación a la categoría de creencias populares, las madres identifican que algunos alimentos no permiten el desarrollo del habla en el niño, mencionando, por ejemplo, el consumo del queso, además, indican que el consumo de pescado, naranja y huevos se encuentra relacionado con alergias y caries. Indican que el crecimiento y desarrollo de niños y niñas es diferente debido a que el niño crece más rápido, come más y es más inquieto y que las niñas se desarrollan más rápido, despiertan antes y que no lactan como deben.

En la categoría de prácticas del cuidado del niño menor de cinco años las madres aplican la consejería del personal de salud en la práctica de la lactancia materna para ellas debe ser exclusiva hasta los 6 meses y se debe dar al niño hasta los 2 años. Además, las madres reconocen que la lactancia materna es importante para el crecimiento y desarrollo del niño, ya que aumenta sus defensas, no siendo necesario el uso de fórmulas. Asimismo, las participantes indican que el cuidado del niño está bajo la responsabilidad de la madre y que el padre ayuda en el cuidado, los familiares como lo abuelos solo brindan el cuidado cuando están de visita.

En relación a las prácticas de la medicina tradicional y medicina convencional refieren prácticas de cuidado frente a enfermedades respiratorias y enfermedades digestivas. Para estas enfermedades, se utiliza la medicina tradicional entre las cuales se encuentran el uso de eucalipto, miel, limón, ruda, quico, orégano, leche materna, saliva, orina, manzanilla, frotación de mentol. Asimismo, indican que se ayudan con la asistencia al establecimiento de salud.

Las limitaciones en el estudio se presentaron por el horario de trabajo y quehaceres de las madres, por lo que las investigadoras debieron realizar citas y realizar las entrevistas en diferentes horarios.

\section{Referencias bibliográficas}

1. Organización Mundial de la Salud. Estrategia mundial para la salud de la mujer, el niño y el adolescente 2016-2030;2016.

2. INEI. Instituto Nacional de Estadística e Informática. Perú: Estimaciones y proyecciones de población por sexo, según departamento, provincia y distrito, 2000-2015;2009.

3. Hernández R. Metodología de la investigación. 5. ${ }^{\text {a }}$ ed. México: Mc Graw Hill; 2016.

4.- Strauss A, y Corbin J. Bases de la investigación cualitativa. Técnicas y procedimientos para desarrollar la teoría fundamentada. Colombia: Editorial Universidad de Antioquia. Facultad de Enfermería de la Universidad de Antioquia; 2002.

5. Marriner, A. y Alligoog M. Modelos y Teorías de Enfermería. 6. ${ }^{\mathrm{a}}$ ed. España: Elsevier Mosby;2008.

6.- Prieto B. Significado que le asigna la madre al cuidado cultural de ella misma y su recién nacido durante el puerperio a partir de sus prácticas y creencias, Universidad Nacional de Colombia; 2012.

7. Leininger M. Cuidados Culturales. Teoría de la diversidad y de la universal. Estados Unidos: New York; 1991.

8. Ministerio de Salud. Análisis de Situación de Salud del Pueblo Aymara. Dirección General de Epidemiologia. Puno-Perú; 2010

9. Camino L. Lactancia una práctica que trasciende los tiempos. Revista Peruana Epidemiológica 1996.9 (2):33-2.

10. Benner P. y Gordon S. Caring practice and caregiving. Philadelphia; 1996, pp.40-55.

11. Harris M. Teorías sobre la cultura en la era posmoderna. Barcelona: Editorial Crítica; 2000.

12. Colliere M. Origen de las prácticas de cuidados, su influencia en la práctica de la enfermería. En: Promover la vida. España: McGraw-Hill Interamericana; 1997.

13. Organización Mundial de la Salud. Medicina Tradicional definiciones. [Internet].2007 [citado Diciembre de 2017]; Disponible en: https://www.who.int/topics/traditional_medicine/d efinitions/es

14. Organización Mundial de la Salud. Fomento y desarrollo de la formación y de las investigaciones en materia de medicina tradicional. Asamblea mundial de la salud. Resolución WHA30.49; 1977 diciembre. Ginebra; 1977

15. Castañeda P. El Lenguaje verbal del niño: ¿cómo estimular, corregir y ayudar para que aprenda a hablar bien? [En línea]. Perú: UNMSM; 1999. . [Citado: 2017 diciembre]. Capítulo 13. El habla y los retrasos en el desarrollo Disponible en: shorturl.at/hsEV7

16. Instituto Nacional de la Sordera y Otros Trastornos de la Comunicación. Etapas del desarrollo del habla y el lenguaje. EE.UU. 2010. 1(4781).

\section{Correspondencia}

karijet@hotmail.com
Fecha de recepción: 04 de octubre 2019

Fecha de aceptación: 22 de noviembre de 2019 